\title{
Selective reaching: evidence for multiple frames of reference
}

Citation for published version (APA):

Keulen, R. F., Adam, J. J. M. E., Fischer, M. H., Kuipers, H., \& Jolles, J. (2002). Selective reaching: evidence for multiple frames of reference. Journal of Experimental Psychology-Human Perception and Performance, 28(3), 515-526. https://doi.org/10.1037/0096-1523.28.3.515

Document status and date:

Published: 01/01/2002

DOI:

10.1037/0096-1523.28.3.515

Document Version:

Publisher's PDF, also known as Version of record

\section{Please check the document version of this publication:}

- A submitted manuscript is the version of the article upon submission and before peer-review. There can be important differences between the submitted version and the official published version of record.

People interested in the research are advised to contact the author for the final version of the publication, or visit the DOI to the publisher's website.

- The final author version and the galley proof are versions of the publication after peer review.

- The final published version features the final layout of the paper including the volume, issue and page numbers.

Link to publication

\footnotetext{
General rights rights.

- You may freely distribute the URL identifying the publication in the public portal. please follow below link for the End User Agreement:

www.umlib.nl/taverne-license

Take down policy

If you believe that this document breaches copyright please contact us at:

repository@maastrichtuniversity.nl

providing details and we will investigate your claim.
}

Copyright and moral rights for the publications made accessible in the public portal are retained by the authors and/or other copyright owners and it is a condition of accessing publications that users recognise and abide by the legal requirements associated with these

- Users may download and print one copy of any publication from the public portal for the purpose of private study or research.

- You may not further distribute the material or use it for any profit-making activity or commercial gain

If the publication is distributed under the terms of Article $25 \mathrm{fa}$ of the Dutch Copyright Act, indicated by the "Taverne" license above, 


\section{Selective Reaching: Evidence for Multiple Frames of Reference}

\author{
Ron F. Keulen and Jos J. Adam \\ Maastricht University
}

\author{
Martin H. Fischer \\ University of Dundee
}

\author{
Harm Kuipers and Jelle Jolles \\ Maastricht University
}

\begin{abstract}
Students participated in 3 experiments investigating the use of environment- and action-centered reference frames in selective reaching. They pointed to a green target appearing either with or without a red distractor. Target-distractor distance was manipulated, and distractor interference (difference between distractor trials and no-distractor trials) was measured in reaction time, movement time, and movement endpoint. Target-distractor distance determined the dominant frame of reference. Small distances evoked an environment-centered framework that encoded targets within an external context. Large distances evoked an action-centered framework that encoded targets relative to the start position of the hand. Results support the hypothesis that the brain represents spatial information in multiple frames of reference, with the dominant frame of reference being dependent on the task demands.
\end{abstract}

A well-known example used to highlight the concept of selective attention is picking a ripe apple from a branch when an unripe apple is nearby on the same branch (e.g., Meegan \& Tipper, 1999). This example shows that selective attention mediates goal-directed action through selection of appropriate actions and inhibition of inappropriate actions (i.e., picking the unripe apple). These selection processes take time, resulting in a longer time to respond to the target when a distractor is present than when it is not. The interfering effect of a distractor on response time, labeled distractor interference, was demonstrated by Tipper, Lortie, and Baylis (1992) in a selective reaching task.

Tipper et al. (1992) asked participants to reach for and depress one of nine target buttons arranged in a $3 \times 3$ matrix. Two small lights, one red (the target) and one yellow (the distractor), were positioned directly below each button. On $20 \%$ of the trials the red light (the target) appeared alone, whereas on the remaining trials the yellow light (the distractor) appeared concurrently with the red light but at a different location. Starting from different home positions, participants had to touch as quickly as possible the button indicated by the red light while ignoring the button associated with the yellow light. Using response time (time from stimulus onset to contact of the target button) as the dependent

Ron F. Keulen, Jos J. Adam, and Harm Kuipers, Department of Movement Sciences, Maastricht University, Maastricht, the Netherlands; Martin H. Fischer, Department of Psychology, University of Dundee, Dundee, Scotland; Jelle Jolles, Department of Neuropsychology and Psychiatry, Maastricht University, Maastricht, the Netherlands.

We thank Scott Glover and two anonymous reviewers for their constructive comments on earlier versions of this article.

Correspondence concerning this article should be addressed to Ron F. Keulen, Department of Movement Sciences, Maastricht University, P.O. Box 616, 6200 MD Maastricht, the Netherlands. E-mail: ron.keulen@bw.unimaas.nl variable, Tipper et al. (1992) found that the presence of a distractor light in the display slowed reaching performance. ${ }^{1}$ Moreover, results showed two asymmetries in the spatial nature of the interference effect. In one, distractors located between the start position of the hand and the position of the target caused greater interference than did distractors located beyond the target. Tipper et al. called this the proximity-to-hand effect. In the other, distractors located in the hemispace ipsilateral to the responding hand caused more interference than did contralateral distractors. Tipper et al. called this the ipsilateral effect. The two effects indicate that the spatial relationship of the target and distractor with the start position of the responding hand is an important determinant of distractor interference.

Following the discovery of distractor interference in selective reaching, Meegan and Tipper (1999) proposed the visuomotor processing hypothesis. This hypothesis attributes interference in the selective reaching task to visuomotor competition from distractors. Distractor interference reflects the need to suppress or inhibit responses toward the distractor. The hypothesis assumes that both target and distractor automatically trigger the planning of movements toward their respective locations. It suggests that the more quickly a reaching movement can be made to a location, the greater the interference from a distractor at that location. This is so because the efficiency of visuomotor processing (planning and execution of movement) depends on the location of the stimulus. The visuomotor processing hypothesis can explain both the proximity-to-hand and ipsilateral effects by assuming that near or ipsilateral locations are associated with shorter reaction or move-

\footnotetext{
${ }^{1}$ Distractor interference effects in selective action are sometimes (e.g., Fischer \& Adam, 2001; Meegan \& Tipper, 1998; Pratt \& Abrams, 1994), but not always (e.g., Castiello, 1996; Jackson, Jackson, \& Rosicky, 1995), replicated. For a discussion of methodological factors possibly contributing to these conflicting results, see Tipper, Howard, and Jackson (1997).
} 
ment times (MTs) than far or contralateral locations, respectively (see also Meegan \& Tipper, 1998).

\section{Spatial Reference Systems}

Encoding a target location implies the existence of a frame of reference in which space coordinates are defined. At least three frames of reference have been proposed for visually guided reaching (e.g., Andersen, Essick, \& Siegel, 1985; Tipper et al., 1992): (a) a viewer-based (or egocentric) frame of reference according to which locations of objects are coded relative to the viewer, (b) an environment-based (or allocentric) frame of reference according to which locations of objects are coded with respect to other objects in the display, and (c) an action-based frame of reference according to which objects are coded relative to the start position of the responding effector. The visuomotor processing hypothesis assumes an action-centered frame of reference (Tipper et al., 1992). That is, selective attention controls goal-directed aiming movements and operates in a spatial domain defined by the relation of the target and distractor positions to the start position of the responding hand. Hence, a given distractor could be more or less distracting, depending on its relation to the hand and target.

The environment-centered framework may play a role in selective reaching as well. Aiming of movements toward targets can also be influenced by the spatial relationships of the target with environmental cues. This point is illustrated by evidence showing that the Ebbinghaus (or Titchener circles) illusion-a target circle surrounded by smaller circles appears to be larger than a target circle surrounded by larger circles-may affect grasping movements (e.g., Franz, Gegenfurtner, Bülthoff, \& Fahle, 2000; but see Haffenden \& Goodale, 2000). Furthermore, Fischer and Adam (2001) reported a spatial repulsion effect indicating that the endpoints of movements were biased away from the location of the distractor, not away from the start position of the responding hand. Thus, the brain may code visual information in multiple frames of reference, with the frame of reference that dominates performance being dependent on the task demands and the nature of the stimuli used (e.g., Allport, 1989; Baylis \& Driver, 1993; Gangitano, Daprati, \& Gentilucci, 1998; Graziano \& Gross, 1998; Tipper, Weaver, \& Houghton, 1994). For instance, the environmentcentered frame of reference, which describes the relationships between or among objects in the environment, might be dominating performance in situations in which target and distractor are close together. In terms of the apple-picking analogy, the issue is whether an unripe apple directly adjacent to a ripe apple causes the same kind of interference as an unripe apple further away. We believe that the answer to this question depends on both bottom-up factors (e.g., target-distractor distance) and top-down factors (e.g., eye fixation strategies). The purpose of the present experiments was to investigate the role of such factors.

So far, most studies have involved rather large distances between target and distractor locations: Tipper and colleagues (Meegan \& Tipper, 1998, 1999; Tipper et al., 1992) used a targetdistractor separation of $13.5 \mathrm{~cm}$, whereas Pratt and Abrams (1994) used a target-distractor separation of $4 \mathrm{~cm}$. These studies revealed substantial proximity-to-hand effects; that is, distractors between hand and target (i.e., near distractors) interfered more than distractors located behind the target (i.e., far distractors). In other words, these studies showed asymmetric interference effects (i.e., unequal amounts of interference for near and far distractors), a key characteristic of action-centered attention. The present study examined the possibility that asymmetric interference effects are limited to conditions that involve relatively large spatial separations between target and distractor, and that very small target-distractor separations might exhibit a symmetric pattern of interference (i.e., similar amounts of interference for near and far distractors). This outcome would be consistent with the use of an environment-centered frame of reference.

In Experiment 1, we used a small target-distractor separation of $5 \mathrm{~mm}$. Our goal was to induce an environment-centered frame of reference. In Experiment 2, we adopted a larger target-distractor separation of $20 \mathrm{~mm}$. Our goal here was to show a proximity-tohand effect and thus the operation of an action-centered frame of reference. In Experiment 3, we used a single target location and systematically manipulated target-distractor separation. Our goal in this experiment was to test the visuomotor processing hypothesis. Overall, our results provide evidence for environmental encoding of target-distractor relationships when target and distractor are close together and dominance of action-centered coding when larger target-distractor separations are used.

\section{Experiment 1: Small Target-Distractor Separation}

In Experiment 1, we examined the pattern of distractor interference in a selective reaching task involving a much smaller targetdistractor separation than that typically used by Tipper and colleagues (Meegan \& Tipper, 1998, 1999; Simone \& Baylis, 1997; Tipper et al., 1992). In addition, the effect of eye position on distractor interference was assessed. We distinguished two eye positions at the moment of stimulus presentation: (a) the eyes-atstart condition, in which the eyes and hand departed from the same start location, and (b) the eyes-at-center condition, in which the eyes and hand departed from different locations, the eyes starting from the center of the display.

We hypothesized that distractor interference would emerge in distinct frames of reference depending on target-distractor separation and eye fixation. We conjectured that a small separation between target and distractor would evoke an environmentcentered rather than an action-centered frame of reference. Hence, we expected a symmetric pattern of interference as well as an effect of eye position. This latter prediction was based on the fact that target and distractor are closer to the fovea when the center of the display is the fixation point than when the start position is the fixation point. Consequently, initial discrimination of target and distractor should be easier in the eyes-at-center condition than in the eyes-at-start condition. This should be especially true when the task requires color discrimination for the distinction between target and distractor (as was the case in the present task), because color is processed in foveal vision. Hence, according to the environmentcentered view, a smaller interference effect should materialize in the eyes-at-center than in the eyes-at-start condition. This prediction is in accordance with recent work by Gentilucci, Daprati, Gangitano, and Toni (1997), who showed that fixating a target object reduces the influence of surrounding cues. In contrast, the action-centered view, with its emphasis on the start position of the hand, predicts no effect of eye position on distractor interference. 


\section{Method}

\section{Participants}

Sixteen students from Maastricht University ( 7 women and 9 men), with a mean age of 22.1 years (range: 18-26), participated. In this and all further experiments, participants were paid the equivalent of about $\$ 5$, were right-handed, had normal or corrected-to-normal visual acuity, and were naive as to the purposes of the experiment.

\section{Apparatus and Stimuli}

Participants were tested individually in a quiet, dimly illuminated room. They were seated on a height-adjustable chair in front of a 43.2-cm (17-in.) video monitor equipped with a touch screen. The monitor was placed in a normal, upright position, and participants made responses with the tip of the right index finger directly on the screen. Participants were positioned such that the body midline was in line with the start box on the left side of the display and the shoulder line (i.e., an imaginary line connecting both shoulders) was parallel to the monitor's screen.

The stimulus display used in the experiment is schematically depicted in Figure 1. The start location and target boxes were presented as squares in white outline on a black background. The target stimulus was presented as a green square completely filling one of the target boxes. The distractor stimulus was presented as a red square completely filling one of the boxes directly adjacent to the target box. From the participant's perspective, the distractor stimulus could appear either to the left or to the right of the target stimulus. However, we use the terms near distractor and far distractor (referring to distractors appearing to the left and to the right of the target, respectively) to describe the location of the distractor in relation to the start position of the hand. All boxes were $10 \mathrm{~mm}$ wide and high. Note, however, that in regard to defining an aiming error, the effective target width was set at $12 \mathrm{~mm}$; this was done to limit the number of errors (i.e., target misses). The five target boxes were arranged in a horizontal array spanning $7 \mathrm{~cm}$, with a distance of $5 \mathrm{~mm}$ between the individual target boxes (side to side). The starting box was located $14 \mathrm{~cm}$ to the left of this array (side to side). The touch screen was interfaced with an MS-DOS pentium computer that controlled stimulus presentation and recorded response times as well as response accuracy. Temporal resolution was $5 \mathrm{~ms}$, and spatial resolution was $0.1 \mathrm{~mm}$

\section{Design}

Participants performed in one single session lasting about $40 \mathrm{~min}$. There were 195 test trials, preceded by 25 practice trials, in each of the two eye position conditions. Order of these conditions was counterbalanced. Within a block of 195 test trials, there were 75 trials without distractors (i.e., 15 for each target) and 120 trials with distractors. For the distractor trials, the three middle targets (i.e., Targets 2, 3, and 4) each included 15 near and 15 far distractors, whereas the first (leftmost) target had 15 far distractors and the last (rightmost) target had 15 near distractors. The order of distractor and no-distractor trials within a block of 195 test trials was random.

\section{Procedure}

At the beginning of each trial, the start box turned green, indicating that the participant could move his or her fingertip to the green start box. In the eyes-at-start condition, contact with the start box caused the green light in the box to disappear and the target stimulus (with or without distractor) to appear and remain on until the response was completed. Participants were informed that, on each trial, a green light would appear in one of the five target boxes and that, on some trials, a red light would appear in a different box. They were instructed to touch the green target box as quickly and

\section{Experiment 1}

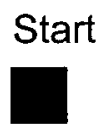

\section{Targets}

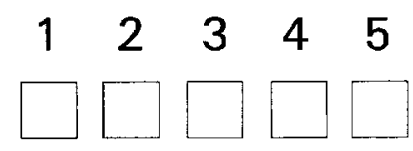

\section{Experiment 2}

\section{Targets}

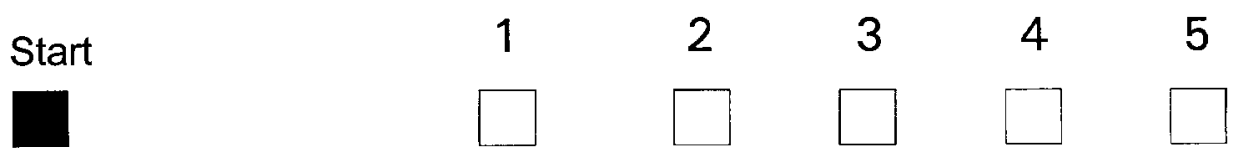

\section{Experiment 3}

Start

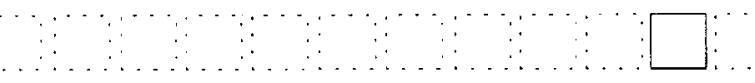

Figure 1. Schematic overview of the visual displays in the three experiments, drawn to scale. The numbers were not actually present. 
accurately as possible while ignoring the red distractor box. If they missed the target, they were encouraged to try to do better in subsequent trials. One second after completion of the aiming response, the start box turned green again, signaling the start of the next trial, which the participants could initiate at will. The computer presented a visual feedback signal if the participant failed to hit the target box or if the start box was released too soon (i.e., within $150 \mathrm{~ms}$ after target presentation). These error messages were, respectively, You missed the target! and Start box released too soon! Also, the computer generated a visual error signal if the participant failed to contact the start box accurately (Depress the start box accurately!). In that case, the trial was repeated.

In the eyes-at-center condition, participants were asked to first contact the start box and then to move their eyes to the center target (Target 3). This shift in gaze position was prompted and facilitated by blinking of the center target three times during a period of 1,000 ms. Then, after an additional delay of $1,000 \mathrm{~ms}$, the target stimulus (with or without distractor) was presented. We calculated two measures of response time: (a) reaction time (RT), measured from the time when the target stimulus appeared to the time when the start box was released, and (b) MT, measured from the time when the start box was released to when the target box was pressed.

\section{Analysis}

RTs below $150 \mathrm{~ms}$ were considered anticipations and were excluded from data analyses; this criterion resulted in the removal of $4.8 \%$ of trials (5.3\% in the eyes-at-start condition and $4.2 \%$ in the eyes-at-center condition). RT, MT, and error percentages were calculated for each participant as a function of eye position, distractor presence, and target location. We performed two different kinds of analyses of variance (ANOVAs). The first, analysis of all targets, included all data and was performed on mean RT, MT, and error percentages; eye position (eyes at start vs. eyes at center), distractor presence (with vs. without distractor), and target location $(1,2,3,4$, or 5$)$ were within-subject variables.

The second analysis, near versus far distractors, compared trials with near and far distractors and involved only the data from the three middle targets (i.e., Targets 2, 3, and 4), because only these targets had both near and far distractors. This analysis was performed on mean RT, MT, and error percentages, and eye position (eyes at start vs. eyes at center) and distractor location (near vs. far distractor) were within-subject variables. We also examined the effect of distractor location (i.e., near vs. far distractor) on the spatial endpoints of the movements. In particular, we assessed biases in the horizontal and vertical dimensions relative to the exact center of the target box in terms of constant error (CE).

Whenever needed, we adjusted tests for heterogeneity of variance and covariances using Huynh-Feldt corrected significance values. We carried out post hoc analyses using Tukey's honestly significant difference procedure; an alpha level of .05 was used to determine statistical significance.

\section{Results}

\section{Analysis of All Targets}

$R T$. There was a main effect of eye position, $F(1,15)=19.80$, $p<.001$, indicating substantially shorter mean RTs in the eyesat-start condition than in the eyes-at-center condition ( 254 vs. 320 $\mathrm{ms}$, respectively). Also, there was a significant Eye Position $\times$ Distractor Presence interaction, $F(1,15)=4.88, p<.05$, indicating a small, nonsignificant $(p>.32)$ amount of distractor interference $(3 \mathrm{~ms})$ in the eyes-at-start condition and a small, nearsignificant $(p<.08)$ amount of distractor facilitation $(5 \mathrm{~ms})$ in the eyes-at-center condition.

MT. Movements tended to be faster in the eyes-at-center condition than in the eyes-at-start condition (428 ms vs. $452 \mathrm{~ms}$ ); however, this effect was not significant, $F(1,15)=2.33, p>.14$. The significant main effect of target position, $F(4,60)=26.77$, $p<.001$, indicated longer MTs for longer distances, except in the case of Target 5, which showed a shorter MT than Target $4(M \mathrm{~s}=$ 421, 434, 445, 454, and $445 \mathrm{~ms}$ for Targets 1, 2, 3, 4, and 5, respectively). This effect of a shorter MT to Target 5 was evident only in the eyes-at-center condition, as indicated by a significant interaction between eye position and target location, $F(4,60)=$ $6.11, p<.001$ (see Figure 2).

The main effect of distractor presence, $F(1,15)=28.36, p<$ .001 , indicated a mean interference effect of $10 \mathrm{~ms}(M \mathrm{~s}=445 \mathrm{vs}$. $435 \mathrm{~ms}$ for the conditions with and without distractors, respectively). However, this interference effect was qualified by an interaction with eye position, $F(1,15)=10.50, p<.01$, indicating less interference in the eyes-at-center condition $(M=5 \mathrm{~ms}, p<$ $.05)$ than in the eyes-at-start condition $(M=15 \mathrm{~ms}, p<.001)$.

Errors. The overall error rate was $8.0 \%$. There was a main effect of eye position, $F(1,15)=6.76, p<.05$, indicating fewer errors in the eyes-at-start condition than in the eyes-at-center condition (6.3\% vs. $9.8 \%$, respectively). This outcome was opposite to the MT data, which showed fastest responses in the eyesat-center condition, suggesting the possibility of a speed-accuracy trade-off. There was also a main effect of target location, $F(4$, $60)=4.42, p<.01$, indicating a larger error rate for Target 5 $(11.2 \%)$ than for the first four targets, which did not differ significantly from each other $(M \mathrm{~s}=7.0 \%, 7.8 \%, 6.7 \%$, and $7.4 \%$, respectively). This outcome was consistent as well with a speedaccuracy trade-off interpretation of the surprisingly fast responses toward Target 5 .

\section{Near Versus Far Distractors}

$R T, M T$, and errors. All $F$ values involving distractor location were nonsignificant (all $p \mathrm{~s}>.1$ ), indicating that near and far distractors had no differential effects on RT, MT, and error rate.

Movement endpoints. In the horizontal dimension, near distractors caused a spatial diversion to the right $(\mathrm{CE}=0.19 \mathrm{~mm})$, far distractors caused a strong deviation to the left $(\mathrm{CE}=-0.67 \mathrm{~mm})$, and trials without distractors caused a modest deviation to the left $(\mathrm{CE}=-0.45 \mathrm{~mm}), F(2,30)=33.44, p<.001$. Note that $\mathrm{CE}$ was measured relative to the center of the target boxes. Thus, this pattern of results indicated that, relative to the neutral no-distractor condition, near distractors caused a spatial bias to the right $(0.64$ $\mathrm{mm})$ and far distractors caused a spatial bias to the left $(-0.22$ $\mathrm{mm})$. Moreover, the significant main effect of eye position, $F(1$, $15)=7.47, p<.05$, indicated a stronger overall deviation to the left (i.e., a larger undershoot) in the eyes-at-start condition than in the eyes-at-center condition (i.e., -0.50 vs. $-0.13 \mathrm{~mm}$, respectively). In the vertical dimension, there were no differential biases, all conditions showing an upward bias (mean $\mathrm{CE}=0.96 \mathrm{~mm}$ ).

\section{Discussion}

Experiment 1 yielded three important outcomes. First, opposite to previous reports, near and far distractors produced similar amounts of interference in the temporal domain. This pattern of symmetric interference effects argues against an action-centered frame of reference and supports an environment-centered frame of reference in which the relation between objects is important. 

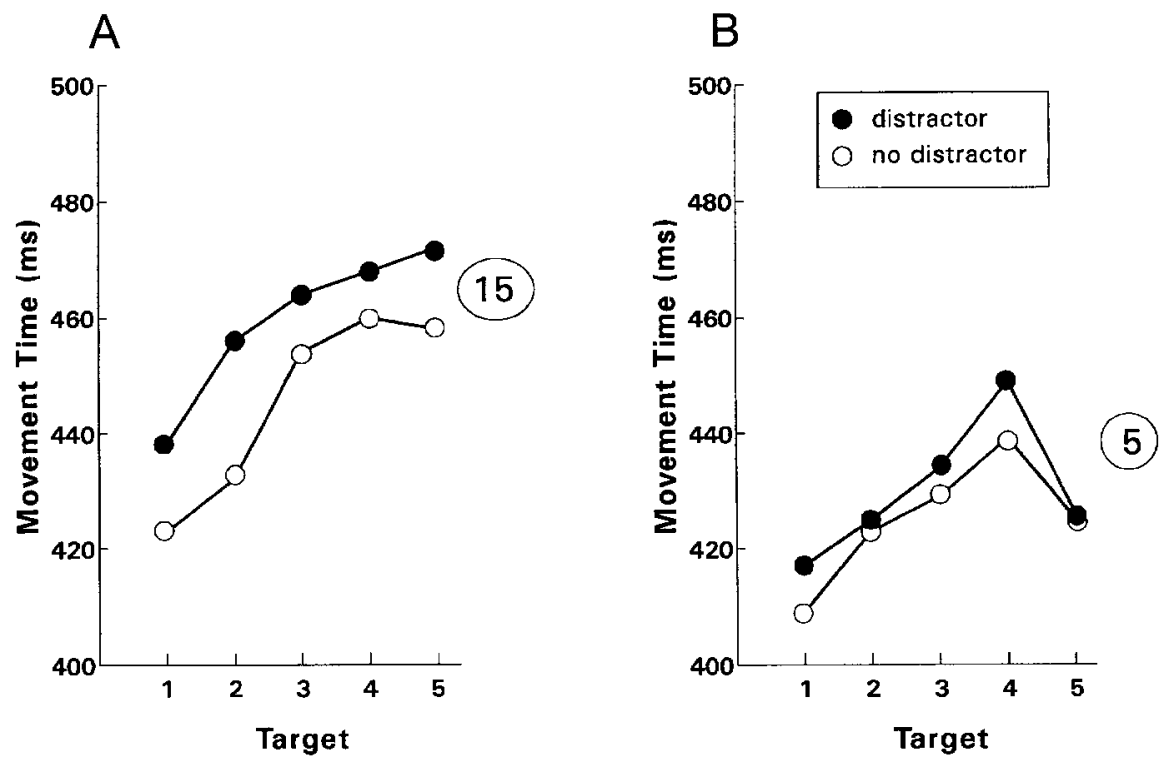

Figure 2. Mean movement time as a function of target position for trials with and without distractors in the eyes-at-start (A) and eyes-at-center (B) conditions in Experiment 1. The circled numbers indicate the amount of movement time interference.

Second, the spatial dispersion of the movement's endpoints indicated that the presence of distractors caused a spatial bias away from the distractor location (and not away from the start position of the hand); this too supports an environment-centered frame of reference. Third, the amount of distractor interference in movement time depended on eye position, such that the eyes-at-center condition produced less interference than the eyes-at-start condition. Our finding of a modulating effect of eye position is compatible with an environment-centered frame of reference, because perceptual discrimination between target and distractor is best achieved with the fovea centered on the display. It is not necessarily the case that point of fixation can interact only with allocentric and not with hand-centered frames. That is, whereas the current work shows that target-directed reaching is affected by point of fixation, Tipper, Howard, and Paul (2001) showed that the path of a saccade is affected by whether a reach to the target is also produced. They interpreted this effect as reflecting cross talk between hand-centered and eye-centered frames (see also Bekkering, Adam, van der Aarssen, Kingma, \& Whiting, 1995).

In summary, the findings of Experiment 1 suggest that spatial separation between target and distractor may be an important mediating variable of distractor interference. As Tipper and colleagues (Meegan \& Tipper, 1998, 1999; Tipper et al., 1992) have shown, large separations evoke an action-centered framework (characterized by asymmetric interference). In contrast, the results of Experiment 1 indicate that small separations may evoke an environment-centered framework (characterized by symmetric interference). We tested this claim further in the next experiment by increasing the separation between target and distractor to $20 \mathrm{~mm}$ in an attempt to demonstrate action-centered attention.

\section{Experiment 2: Large Target-Distractor Separation}

In Experiment 2, we used a larger spatial separation between target and distractor than in Experiment 1 (i.e., $20 \mathrm{~mm}$ instead of
$5 \mathrm{~mm}$ ). According to the present hypothesis, this manipulation should induce the use of an action-centered reference system instead of an environment-centered one. This in turn should lead to an asymmetric interference effect (the proximity-to-hand effect) that is independent of eye position.

\section{Method}

\section{Participants}

Sixteen new students ( 6 women and 10 men) from the same subject pool as in Experiment 1 participated. Mean age was 22.2 years (range: 19-25).

\section{Materials and Apparatus}

The five target boxes were arranged in a horizontal array spanning 13 $\mathrm{cm}$, with a distance of $20 \mathrm{~mm}$ between the individual target boxes (side to side). The starting box was located $8 \mathrm{~cm}$ to the left of this array (see Figure 1). Otherwise, the materials and apparatus were the same as in Experiment 1.

\section{Design and Procedure}

The design and procedure were the same as in Experiment 1.

\section{Analysis}

The Experiment 1 criterion for exclusion of trials resulted in the removal of $4.3 \%$ of the trials $(5.3 \%$ in the eyes-at-start condition and $3.3 \%$ in the eyes-at-center condition).

\section{Results}

\section{Analysis of All Targets}

$R T$. There was a main effect of eye position, $F(1,15)=10.41$, $p<.01$, indicating, as in Experiment 1, shorter mean RTs in the 
eyes-at-start condition than in the eyes-at-center condition ( $258 \mathrm{vs.}$ $296 \mathrm{~ms}$, respectively). Also, there was a main effect of target position, $F(4,60)=3.85, p<.01$, indicating that RT to the first target was significantly longer than RT to the other four targets, which did not differ significantly from each other $(284,276,277$, 275 , and $275 \mathrm{~ms}$ for left to right targets, respectively).

MT. As in Experiment 1, movements in the eyes-at-center condition tended to be executed faster than movements in the eyes-at-start condition ( $450 \mathrm{~ms}$ vs. $470 \mathrm{~ms}$ ), but, again, this effect was not significant, $F(1,15)=1.73, p>.2$. The main effect of target position, $F(4,60)=136.84, p<.001$, indicated longer MTs for longer distances $(M \mathrm{~s}=403,433,460,487$, and $515 \mathrm{~ms}$ for left to right targets, respectively). The significant Target Position $\times$ Eye Position interaction, $F(4,60)=3.76, p<.01$, indicated that the function relating MT to target location (i.e., target distance) was somewhat steeper for the eyes-at-start condition than for the eyes-at-center condition (see Figure 3).

The main effect of distractor presence, $F(1,15)=7.75, p<.05$, indicated a small $(5 \mathrm{~ms})$ but significant amount of interference (462 vs. $457 \mathrm{~ms}$ for trials with and without distractors, respectively). This interference effect did not interact with eye position, $F(1,15)<1$.

Errors. The overall error rate was $10.9 \%$. There was a main effect of eye position, $F(1,15)=4.96, p<.05$, with fewer errors for the eyes-at-start position than for the eyes-at-center position ( $9.6 \%$ and $12.1 \%$, respectively). The fact that the eyes-at-start condition also tended to produce slower movements than the eyes-at-center condition suggests the possibility of a speedaccuracy trade-off. Finally, the main effect of target location, $F(4$, $60)=8.41, p<.001$, indicated an increasing number of errors for targets located further away $(M \mathrm{~s}=8.0 \%, 9.6 \%, 9.4 \%, 11.5 \%$, and $15.9 \%$, respectively).

\section{Near Versus Far Distractors}

$R T, M T$, and errors. Only the ANOVA with MT as the dependent variable yielded a significant main effect of distractor location, $F(1,15)=6.27, p<.05$, indicating interference for near but not for far distractors ( $M \mathrm{~s}=8 \mathrm{vs} .1 \mathrm{~ms}$, respectively). All other $F$ values involving distractor location were nonsignificant (all $p s>.11$ ), indicating that there was no effect of distractor location on RT and error rate.

Movement endpoints. In the horizontal dimension, near distractors caused a substantial spatial diversion to the right $(\mathrm{CE}=$ $0.33 \mathrm{~mm}$ ), whereas far distractors and trials without distractors caused only minor spatial deviations (CEs $=0.07$ and $0.06 \mathrm{~mm}$, respectively), $F(2,30)=2.94, p<.09$. This pattern of results indicated that, relative to the neutral no-distractor condition, near distractors tended to produce a modest spatial shift to the right $(0.27 \mathrm{~mm})$, whereas far distractors caused no spatial bias. In the vertical dimension, there were no differential biases, all conditions showing an upward bias (mean $\mathrm{CE}=0.86 \mathrm{~mm}$ ).

\section{Discussion}

Experiment 2 involved a larger target-distractor separation than Experiment 1. Consistent with the predictions of the actioncentered view, results showed a proximity-to-hand effect (slower responses for near than for far distractors, along with a spatial bias away from near distractors only) that was independent of eye position. This outcome deviates markedly from the results of Experiment 1 (symmetric interference and a modulating effect of eye position on the amount of interference) but is consistent with results of previous studies of distractor interference (e.g., Tipper et al., 1992). Taken together, the results of Experiments 1 and 2 provide evidence for a mediating role of target-distractor separation in distractor interference.
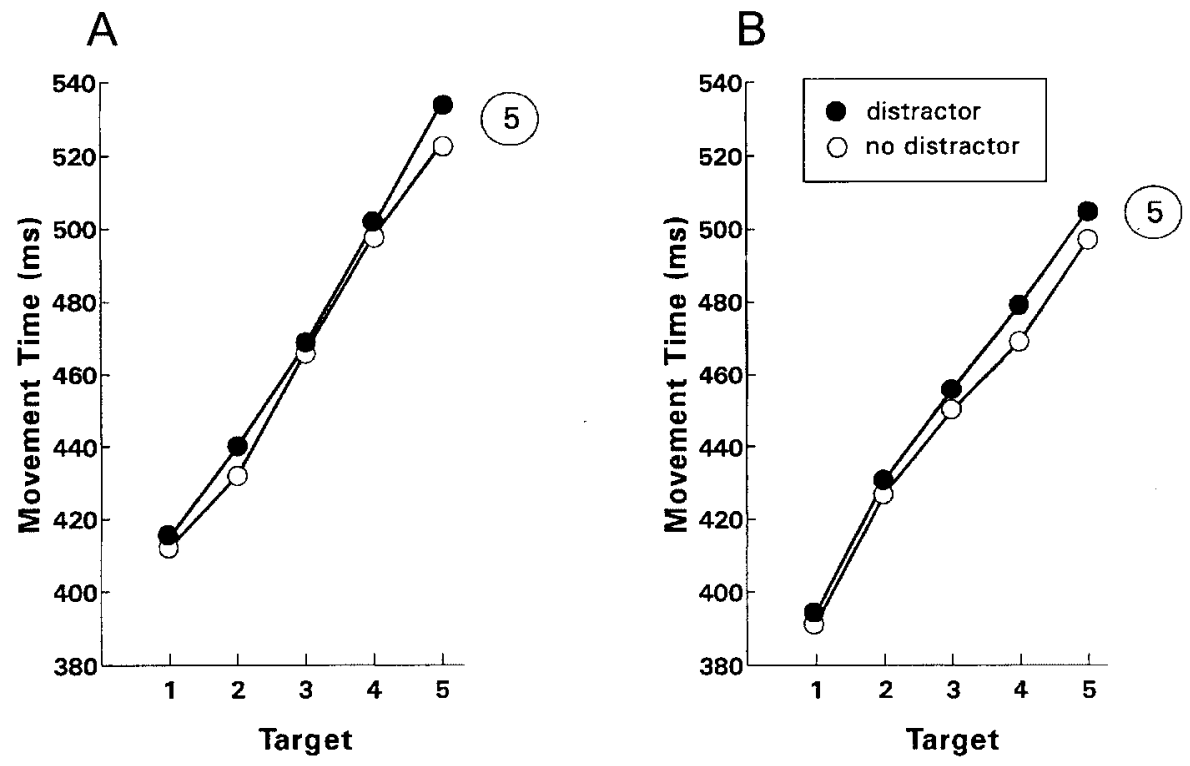

Figure 3. Mean movement time as a function of target position for trials with and without distractors in the eyes-at-start (A) and eyes-at-center (B) conditions in Experiment 2. The circled numbers indicate the amount of movement time interference. 
To substantiate this claim statistically, we performed two between-experiments analyses using MT as the dependent variable. In the first ANOVA, distractor presence (with vs. without distractor), eye position (eyes at start vs. eyes at center), and target location (Target 1, 2, 3, 4, or 5) were within-subject variables, and target-distractor separation ( 5 vs. $20 \mathrm{~mm}$ ) was a between-subjects variable. This analysis revealed a Target-Distractor Separation $\times$ Eye Position $\times$ Distractor Presence interaction, $F(1,30)=5.64$, $p<.05$, indicating that amount of distractor interference was a joint function of target-distractor separation and eye position. That is, with the small target-distractor separation there was more interference in the eyes-at-start condition than in the eyes-at-center condition, whereas with the large target-distractor separation distractor interference was independent of eye position.

In the second ANOVA, distractor location (near vs. far distractor), eye position (eyes at start vs. eyes at center), and target location (Target 2, 3, or 4) were within-subject variables, and target-distractor separation ( $5 \mathrm{vs} .20 \mathrm{~mm}$ ) was a between-subjects variable. This analysis revealed a Target-Distractor Separation $\times$ Distractor Location interaction, $F(1,30)=7.67, p<.01$, indicating symmetry and asymmetry of near and far distractors for small and large target-distractor separations, respectively. This interaction is depicted graphically in Figure 4A and Figure 4B.

The first between-experiments analysis also revealed another important interaction: Target-Distractor Separation $\times$ Target Location, $F(4,120)=57.88, p<.001$. This interaction, graphically depicted in Figure 5 (left), showed a different MT pattern across target locations in Experiment 1 (small intertarget distance) than in Experiment 2 (large intertarget distance). Specifically, MT exhibited the usual linear relationship with movement distance for large but not for small intertarget distances, the latter showing a small decrease in MT for Target 5 relative to Target $4 .^{2}$

Interestingly, and in contrast to our previous suggestion, this unusual finding might not be due to a speed-accuracy trade-off phenomenon, in that the larger error rate for Target 5 was also

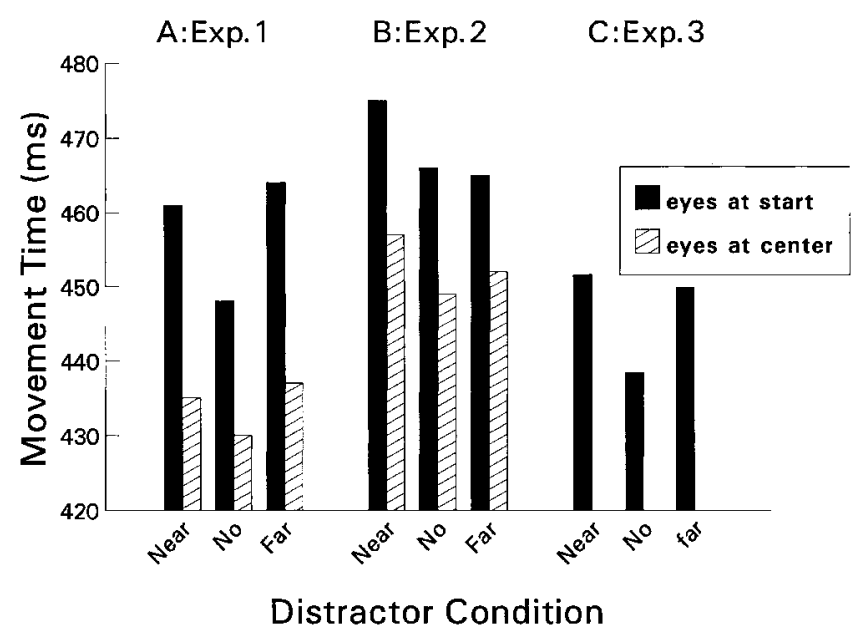

Figure 4. Mean movement time as a function of distractor condition and eye position in Experiments 1 (A), 2 (B), and 3 (C). In Experiment 3, the movement time values for the near and far distractor conditions were averaged across all target-distractor distances. Exp. = experiment; Near = near distractor; No = no distractor; Far $=$ far distractor. found in the large intertarget distance condition (see right panel of Figure 5). That is, in terms of error rates, there was no interaction between intertarget distance and target location, $F(4,120)=1.28$, $p>$.23. From this result, it appears that the relatively short MTs for Target 5 in Experiment 1 cannot be attributed to a trade-off between speed and accuracy.

An alternative explanation focuses on the idea that the small intertarget distances in Experiment 1 may have led to a perceptual grouping or clustering of the individual targets. This idea is prompted by evidence showing that preattentive organizational factors, such as those embodied in Gestalt principles, strongly mediate visual selection processes. That is, visual attention operates on structural units or perceptual groups derived from an early, hierarchical segmentation of the visual field according to Gestalt principles (e.g., Duncan, 1984; Logan, 1996; Yantis, 1992). This perceptual grouping account may explain the present inverted-U function, because outer elements in a group - the end positionsappear to have an advantage in terms of response speed (e.g., Adam, 1994; Adam et al., 1998). Note that this account is consistent with an environment-centered frame of reference highlighting the relationship of objects to each other.

RT was shorter in the eyes-at-start condition than in the eyesat-center condition. This phenomenon was present both in Experiment 2 and in Experiment 1 and therefore should not have hampered the between-experiments comparison. It seems plausible that this phenomenon was caused by the greater temporal predictability of target onset in the eyes-at-start condition than in the eyes-at-center condition. In the eyes-at-start condition the target appeared as soon as the start box was touched, whereas in the eyes-at-center condition an interval of $2 \mathrm{~s}$ elapsed between contact with the start box and presentation of the target.

Finally, in Experiment 2 the distractors influenced MT only. This finding does not necessarily contradict the visuomotor processing hypothesis-which attributes distractor interference to an early response selection stage-because the target always appeared to the right of the start location. Thus, the initial movement direction was constant throughout the experiment, allowing target selection to occur after movement initiation.

\section{Experiment 3: Manipulating Target-Distractor Separation}

The results of the first two experiments indicated that, depending on the separation between target and distractor, interference effects originate in an action- or environment-centered framework. According to the visuomotor processing hypothesis, which assumes an action-centered view, interference accrues in a very early stage in the response selection process (Meegan \& Tipper, 1998, 1999). This notion is supported by evidence indicating that when the position of the target is known in advance, distractor interference disappears (e.g., Jackson, Jackson, \& Rosicky, 1995; Tipper,

\footnotetext{
${ }^{2}$ Note that, on average, the movement amplitude (i.e., distance from start position to target) was shorter in Experiment 2 than in Experiment 1. To remove this potential confound, we conducted an additional analysis that included only those targets that had the same distance to the start location in both experiments (i.e., Targets 1,3, and 5 in Experiment 1 and Targets 3, 4, and 5 in Experiment 2). This analysis too yielded a highly significant Target-Distractor Separation $\times$ Target Location interaction, $F(2,60)=6.27, p<.01$.
} 

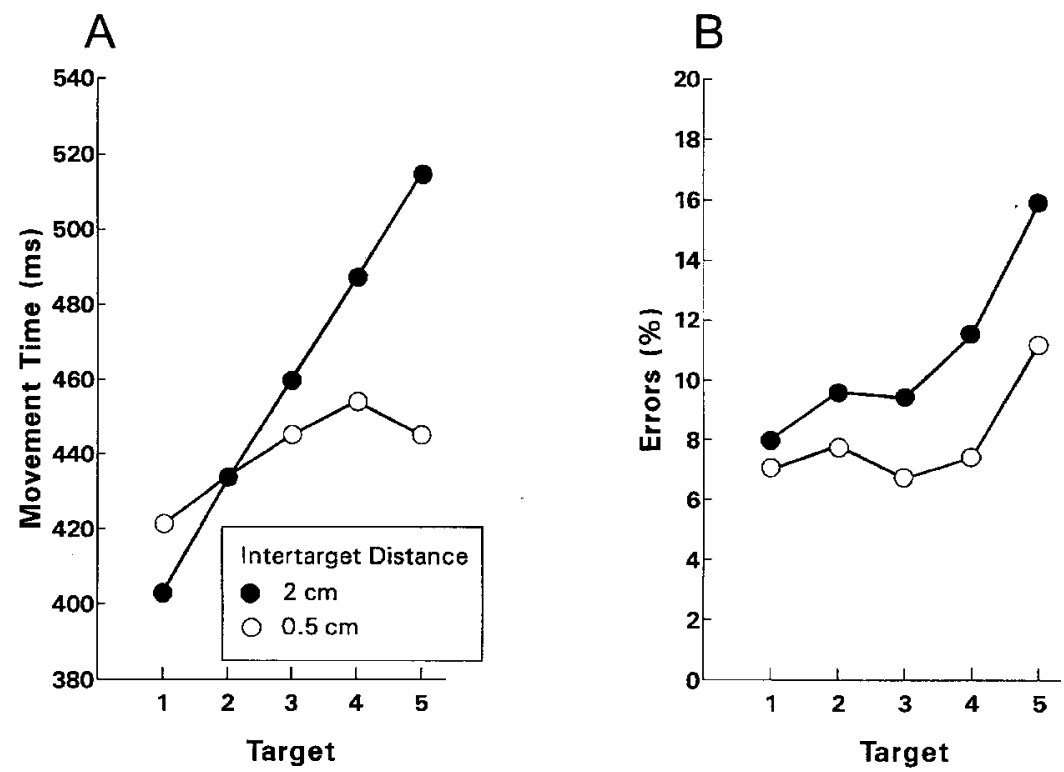

Figure 5. Mean movement time (A) and error percentage (B) as a function of target position and intertarget distance.

Howard, \& Jackson, 1997). According to the visuomotor processing hypothesis, this occurs because advance information regarding the position of the target allows selection of the motor representation of the target and inhibition of the motor representation of the competing distractor before observable behavior begins. Note, however, that this evidence was obtained in experiments involving large target-distractor separations (i.e., 10 and $26 \mathrm{~cm}$ in the Jackson et al., 1995, and Tipper et al., 1997, studies, respectively).

The goal of the present experiment was to determine whetherand, if so, how-small target-distractor separations would influence movements to a fixed target location. According to the visuomotor processing hypothesis, which assumes an actioncentered framework, no interference should be observed. In contrast, if small target-distractor separations evoke environmentcentered representations, substantial and symmetric interference might be expected. This is because environment-centered representations are assumed to play a role throughout the movement production process, that is, not only during initial response planning and selection but also later, in the final stages, at which homing-in processes guide the hand to the final target location. Furthermore, gradually increasing target-distractor separation should lead to progressively weaker effects, as it is well established that interference effects decrease with increasing targetdistractor separation (e.g., Eriksen \& Eriksen, 1974).

On the basis of these premises, we used only one target location in Experiment 3 and systematically varied the distance between the target and distractor. According to the environment-centered view, near and far distractors should show similar amounts of interference and, moreover, should show smaller interference effects for larger distances between target and distractor. According to the visuomotor processing hypothesis, which assumes an actioncentered view, there should be no interference.

\section{Method}

\section{Participants}

Eighteen new students (10 women and 8 men) from the same subject pool as in Experiment 1 participated. Mean age was 23.1 years (range: 18-34).

\section{Materials and Apparatus}

The target was a green square $(10 \times 10 \mathrm{~mm})$ that always appeared $12 \mathrm{~cm}$ to the right of the start location (side to side). The distractor was a red square $(10 \times 10 \mathrm{~mm})$ that could appear either to the left (near) or to the right (far) of the target at one of 10 possible target-distractor distances (side to side): $2,14,26,38,50,62,74,86,98$, or $110 \mathrm{~mm}$. To constrain the degree of variability in the data, we reduced the number of targetdistractor distances from 10 to 5 . That is, we distinguished five new target-distractor conditions by averaging the first two distances, the second two distances, and so forth. This resulted in five target-distractor conditions with mean distances of 8, 32, 56, 80, and $104 \mathrm{~mm}$ to the target. Otherwise, the materials and apparatus were the same as in Experiment 1.

\section{Design and Procedure}

Participants performed in a single session lasting about $30 \mathrm{~min}$. There were 360 test trials preceded by 25 practice trials. Within the block of 360 test trials, there were 60 trials without distractors and 300 with distractors. For each of the 10 possible distractor locations at both sides, there were 15 trials. Order of distractor trials and no-distractor trials was random. The eyes-at-start procedure of Experiment 1 was used. Otherwise, the design and procedure were the same as in Experiment 1.

\section{Analysis}

The Experiment 1 criterion resulted in the removal of $5.7 \%$ of the trials. 


\section{Results}

\section{Effect of Distractor Presence}

Trials with distractors resulted in significantly longer MTs than trials without distractors, $F(1,17)=16.89, p<.001(M \mathrm{~s}=450$ vs. $438 \mathrm{~ms}$, respectively). However, there was no effect of distractor presence on RT, $F(1,17)<1(M \mathrm{~s}=262$ and $262 \mathrm{~ms}$, respectively), or error rate, $F(1,17)<1(M \mathrm{~s}=2.6 \%$ and $2.6 \%$, respectively).

\section{Effect of Distractor Location}

Movement time. We submitted mean interference scores (i.e., difference scores between the control no-distractor condition and the various distractor conditions) to a $2 \times 5$ ANOVA with distractor location (near vs. far) and target-distractor distance $(8,32$, 56,80 , or $104 \mathrm{~mm}$ ) as within-subject variables. Only one significant effect materialized, namely the main effect of targetdistractor distance with MT interference as the dependent variable, $F(4,68)=3.25, p<.05$. This effect indicated that distractors adjacent to the target interfered more than distractors further away from the target, reflecting a gradient of interference around the target (see Figure 6). The $F$ tests involving distractor location were nonsignificant ( $p s>.61$ ), indicating similar amounts of interference for near and far distractors (see also Figure 4C).

Movement endpoints. We calculated mean interference scores by subtracting the mean $\mathrm{CE}$ of the various distractor conditions from the mean $\mathrm{CE}$ of the no-distractor trials. These interference scores were submitted to a $2 \times 5$ ANOVA with distractor location (near vs. far) and target-distractor distance (8, 32, 56, 80, or 104 $\mathrm{mm})$ as within-subject variables. In the horizontal dimension, there was a significant main effect of distractor location, $F(1,17)=$ $5.17, p<.05$, indicating that, on average, near distractors caused a bias to the right $(0.23 \mathrm{~mm})$ and far distractors caused a bias to the left $(-0.33 \mathrm{~mm})$. The significant interaction between distractor location and target-distractor distance, $F(4,68)=7.05, p<.001$, followed up by simple effects tests, indicated that this spatial

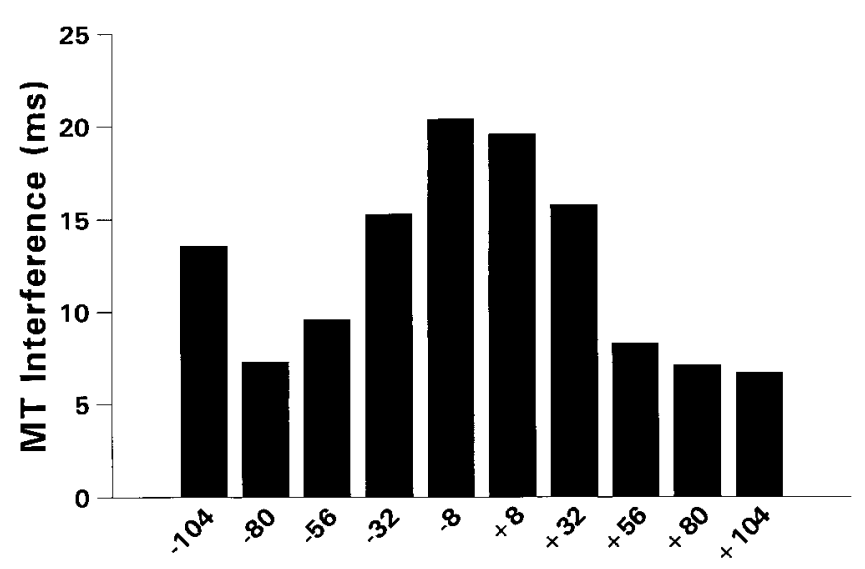

Target-Distractor Separation $(\mathrm{mm})$

Figure 6. Mean movement time (MT) interference as a function of target-distractor separation in Experiment 3. Negative and positive values denote near and far distractors, respectively.

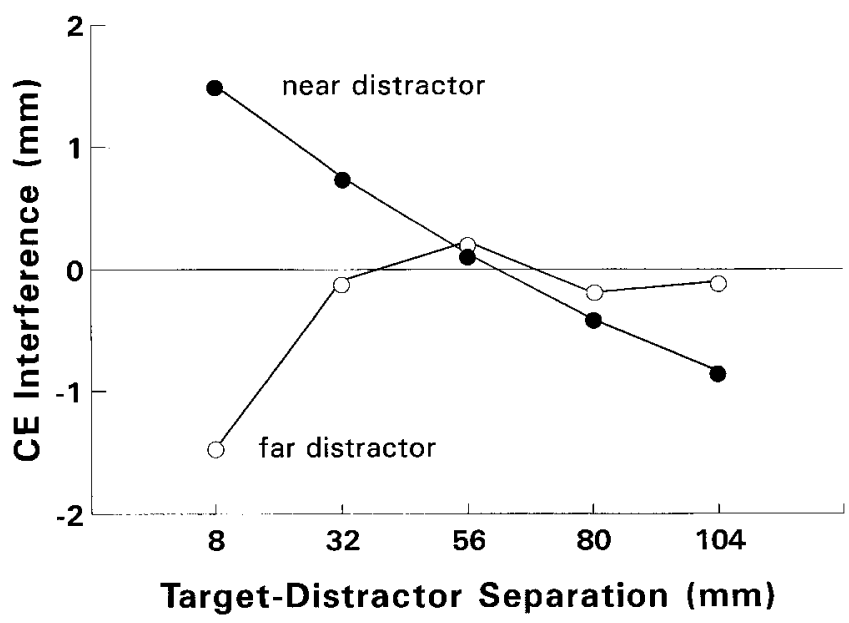

Figure 7. Mean constant error (CE) interference as a function of targetdistractor separation for near and far distractors in Experiment 3. Positive abscissa values denote rightward displacements, whereas negative values denote leftward displacements.

repulsion effect materialized only for the smallest target-distractor separation of $8 \mathrm{~mm}$ (see Figure 7). In the vertical dimension, there were no differential biases, all conditions showing an upward bias (mean CE $=1.19 \mathrm{~mm}$ ).

\section{Discussion}

Throughout the experiment, there was only one fixed target location, and participants made 360 movements to this target with or without distractors presented at varying distances in front of (near) or behind (far) the target. The results showed a symmetric pattern of distractor interference. There were similar amounts of interference for distractors presented at equal distances from the target but at unequal distances from the start position of the hand. This outcome supports an environment-centered reference frame. According to the visuomotor processing hypothesis, early movement selection processes should have been minimal under the present circumstances, and hence no interference effects were expected. The fact that strong interference effects did show up is incompatible with the visuomotor processing hypothesis.

Nevertheless, although statistically not reliable, there seemed to be a hint in the data of a proximity-to-hand effect. That is, distractors closest to the hand's start position tended to show more interference than distractors at the corresponding position behind the target (see Figure 6). Similarly, near distractors close to the target pushed the movement endpoints to the right (i.e., away from the distractor), whereas near distractors close to the hand (start location) appeared to push the movement endpoints to the left (i.e., toward the distractor; see Figure 7). These effects might be interpreted in terms of a proximity-to-hand effect suggesting the possibility that action-centered and environment-centered reference frames may coexist, jointly shaping visuomotor behavior. This possibility is in line with recent evidence suggesting that target position is coded in terms of a weighted contribution of egocentric and allocentric information (Gangitano et al., 1998; Gentilucci, Chieffi, Daprati, Saetti, \& Toni, 1996). 
Finally, the results showed that distractor interference was stronger with close target-distractor spacing than with wide spacing. This outcome is compatible with the well-established distance effects in the flanker paradigm, showing the dependence of flanker interference on target-flanker separation (e.g., Eriksen \& Eriksen, 1974; Miller, 1991). Nevertheless, the relatively large targetdistractor distances of 32 and $56 \mathrm{~mm}$ produced amounts of (symmetric) interference that, although substantially reduced, were still significant. This finding might seem remarkable in light of the results of Experiments 1 and 2 showing a transition of environment-centered to action-centered frameworks with an increase in target-distractor separation from 5 to $20 \mathrm{~mm}$. Perhaps this outcome was related to the much smaller error rate in the present experiment (2.6\%) than in Experiments 1 and 2 (i.e., $8.0 \%$ and $10.9 \%$, respectively), suggesting the possibility of an extended decelerative or homing-in phase-and thus more accurate movements-in the present experiment relative to the first two experiments.

\section{General Discussion}

In contrast to the rich and strong neurophysiological and behavioral evidence for many different egocentric spatial coding systems (including retina-, head-, trunk-, shoulder-, and hand-centered coding systems), the evidence for systems that code object location with respect to the location of other objects is more limited (Woodin \& Allport, 1998). The present results provide evidence for just this latter kind of environment-centered coding in a pointing task in which potential objects are close together (Experiment 1). In these circumstances, near and far distractors caused symmetric interference effects, that is, similar amounts of response slowing and a spatial repulsion effect that pushed the movement endpoints away from the distractor location rather than away from the start position of the hand. In contrast, Experiment 2, which involved larger separations between the relevant objects in the display, showed an asymmetric pattern of interference effects, replicating previous reports and suggesting the use of an actioncentered frame of reference (e.g., Tipper et al., 1992). ${ }^{3}$ In addition, the inverted-U function relating MT to target position found with the small intertarget distance in Experiment 1 deviated markedly from the usual linear function found with the large intertarget distance in Experiment 2. Taken together, these results establish a double dissociation between reaching to a clustered group of objects and reaching to more widely spaced objects and suggest a functional separation between action- and environment-centered spatial coding systems in selective reaching.

However, one might object to this view by claiming that the present data can also be accommodated by a simpler, singleframework account. For example, the symmetric interference effects with small distances might also be explained in terms of an action-centered model assuming that the effects on movement time were inversely proportional to the distance between the distractor and the start location of the hand. Given this assumption, one would expect an asymmetric effect for large distances between target and distractor. This is because in the far condition (i.e., when the distractor is located behind the target) the distractor is too far from the start position of the hand to have an effect, whereas in the near condition (i.e., when the distractor is located between the starting position of the responding hand and the target) the dis- tractor is still close to the start position of the hand. Conversely, one would expect a symmetric effect for small distances, because the distractor then has relatively similar distances to the start position of the hand in the far and near conditions. Even though we agree that the MT interference data might be interpreted in terms of such a unified action-centered model, we believe that the spatial repulsion data are at odds with it because they show unambiguously that the landing points were biased away from the distractor location and not away from the initial hand position. That is, the spatial repulsion data showed a directional effect (and not merely a range effect) that was tied to the position of the distractor rather than to the (start) position of the responding effector. This outcome, in our view, is incompatible with a unified, action-coding account of the present results.

\section{Spatial Repulsion Effect}

Movement endpoints were biased away from the distractor's location. This spatial repulsion effect-first reported by Fischer and Adam (2001) — adds to previous reports showing hand path deviations caused by the presence of distractors (e.g., Howard \& Tipper, 1997). Interestingly, however, hand paths may veer either away or toward distractors (e.g., Tipper et al., 1997; Welsh, Elliott, \& Weeks, 1999). Tipper and colleagues postulated a tentative neurophysiological model to explain hand path deviations (Howard \& Tipper, 1997; Tipper, Howard, \& Houghton, 2000; Tipper et al., 1997). In essence, their model is based on the idea of population coding in the human cortex (e.g., Georgopoulos, 1990), and path interference effects are explained in terms of a selection process from overlapping population codes-activated by the target and distractor-through inhibitory mechanisms. Because the final endpoint of a movement is often closely related to its path, the population coding model might also apply to the spatial repulsion effect.

\section{Eye Position}

Experiments 1 and 2 involved two different eye position instructions: eyes at start and eyes at center. Because an eye tracking apparatus was not available, the experimenter carefully monitored throughout the experiment whether the participants followed the eye movement instructions. It appeared that all participants easily complied with these instructions. This was not surprising, because we implemented two procedural factors that commanded in a natural way the desired eye movement behavior. In the eyes-atstart condition, the start box was so small (i.e., $1 \times 1 \mathrm{~cm}$ ) that eye fixation was necessary to hit it (an error signal was generated if participants failed to do so). In the eyes-at-center condition, after the participant made contact with the start box, the center target blinked three times during a period of $1,000 \mathrm{~ms}$ (followed by an additional delay of 1,000 ms before the target stimulus appeared).

\footnotetext{
${ }^{3}$ A note of caution concerns the validity of asymmetric interference as evidence for an action-centered frame of reference in Experiment 2. Unambiguous evidence would necessitate a manipulation of hand position followed by the demonstration that the asymmetric interference profile is tied to the position of the hand. Tipper et al. (1992) did find a pattern of asymmetric distractor interference that depended on the position of the hand, consistent with an action-centered framework.
} 
Because the blinking box created a series of sudden onsets-which are known to automatically attract attention-it was natural for the participants to move their eyes to the cued center box. And this is what participants did, as checked by the experimenter and furthermore bolstered by the MT data, which showed different functions relating MT to target position in the two eye position conditions, suggesting a functional distinction between the eyes-at-start and eyes-at-center conditions (see Figures 2 and 3).

\section{Locus of Distractor Interference}

According to the visuomotor processing hypothesis, which assumes an action-centered view, distractor interference accrues when objects are first perceived and reflects early motor selection processes (Meegan \& Tipper, 1998, 1999). Complementary to this view, it is also possible that interference effects arise later in the movement production process, that is, after the initial selection processes have been completed and when visual-feedback-based corrective processes are needed to guide the limb to the exact target location.

This possibility is consistent with evidence showing that accurate aiming movements typically have two components: a ballistic, distance covering phase that brings the limb to the vicinity of the target followed by a corrective (current-control or homing-in) phase that ensures accurate movement termination on the target through feedback-based corrective adjustments (e.g., Meyer, Abrams, Kornblum, Wright, \& Smith, 1988; Rosenbaum, 1991). Thus, there may be at least two selection problems to solve when one is reaching to a target that is closely flanked by a distractor: (a) determining the general location of the target and distractor and (b) distinguishing the specific location of the target relative to the distractor. The first problem arises relatively early (i.e., during response planning) and may benefit in particular from an actioncentered framework, because the critical task is to move the hand over a relatively large distance to the target area. The second problem arises later (i.e., during response execution) and may benefit especially from an environment-centered framework, because the critical task is to terminate the movement accurately at a specific object (i.e., hit the target and not the distractor). Of course, this is not to say that environment-centered coding might not play a role in the earlier stages concerned with response planning and initial response selection. Future experimentation, examining movement kinematics and trajectories, should examine this issue.

\section{Conclusion}

In selective reaching, distractors are potential targets and, as such, can compete for control of action; this competition influences reaching movements to the actual target (e.g., Meegan \& Tipper, 1998). The present study suggests that distractor competition may take place in different spatial coordinate systems, with distance between target and distractor being an important mediating variable. This conclusion accords with the hypothesis that the brain represents spatial information in multiple reference frames, with the reference frame dominating performance being dependent on task demands.

\section{References}

Adam, J. J. (1994). Manipulating the spatial arrangement of stimuli in a spatial precuing task. Acta Psychologica, 85, 183-202.
Adam, J. J., Paas, F. G. W. C., Teeken, J. C., van Loon, E., van Boxtel, M. P. J., Houx, P. J., \& Jolles, J. (1998). Effects of age on performance in a finger precuing task. Journal of Experimental Psychology: Human Perception and Performance, 24, 870-883.

Allport, D. A. (1989). Visual attention. In M. I. Posner (Ed.), Foundations of cognitive science (Vol. 2, pp. 631-682). Cambridge, MA: MIT Press.

Andersen, R. A., Essick, G. K., \& Siegel, R. M. (1985, October 25). Encoding of spatial location by posterior parietal neurons. Science, 230, $456-458$.

Baylis, G. C., \& Driver, J. (1993). Visual attention and objects: Evidence for the hierarchical coding of location. Journal of Experimental Psychology: Human Perception and Performance, 19, 451-470.

Bekkering, H., Adam, J. J., van der Aarssen, A., Kingma, H., \& Whiting, H. T. A. (1995). Interference between saccadic eye and goal-directed hand movements. Experimental Brain Research, 106, 475-484.

Castiello, U. (1996). Grasping a fruit: Selection for action. Journal of Experimental Psychology: Human Perception and Performance, 22, 582-603.

Duncan, J. (1984). Selective attention and the organization of visual information. Journal of Experimental Psychology: General, 113, 501517.

Eriksen, B. A., \& Eriksen, C. W. (1974). Effects of noise letters upon the identification of a target letter in a nonsearch task. Perception \& Psychophysics, 16, 143-149.

Fischer, M. H., \& Adam, J. J. (2001). Distractor effects in pointing: The role of spatial layout. Experimental Brain Research, 136, 507-513.

Franz, V. H., Gegenfurtner, K. R., Bülthoff, H. H., \& Fahle, M. (2000). Grasping visual illusions: No evidence for a dissociation between perception and action. Psychological Science, 11, 20-25.

Gangitano, M., Daprati, E., \& Gentilucci, M. (1998). Visual distractors differentially interfere with the reaching and grasping components of prehension movements. Experimental Brain Research, 122, 441-452.

Gentilucci, M., Chieffi, S., Daprati, E., Saetti, M. C., \& Toni, I. (1996). Visual illusion and action. Neuropsychologia, 34, 369-376.

Gentilucci, M., Daprati, E., Gangitano, M., \& Toni, I. (1997). Eye position tunes the contribution of allocentric and egocentric information to target localization in human goal-directed arm movements. Neuroscience Letters, 222, 123-126.

Georgopoulos, A. P. (1990). Neurophysiology of reaching. In M. Jeannerod (Ed.), Attention and performance XIII: Motor representation and control (pp. 227-263). Hillsdale, NJ: Erlbaum.

Graziano, M. S. A., \& Gross, C. G. (1998). Spatial maps for the control of movement. Current Opinion in Neurobiology, 8, 195-201.

Haffenden, A. M., \& Goodale, M. A. (2000). Independent effects of pictorial displays on perception and action. Vision Research, 40, 15971607.

Howard, L. A., \& Tipper, S. P. (1997). Hand deviations away from visual cues: Indirect evidence for inhibition. Experimental Brain Research, $113,144-152$.

Jackson, S. R., Jackson, G. M., \& Rosicky, J. (1995). Are non-relevant objects represented in working memory? The effect of non-target objects on reach and grasp kinematics. Experimental Brain Research, 102, $519-530$.

Logan, G. D. (1996). The CODE theory of visual attention: An integration of space-based and object-based attention. Psychological Review, 103, 603-649.

Meegan, D. V., \& Tipper, S. P. (1998). Reaching into cluttered visual environments: Spatial and temporal influences of distracting objects. Quarterly Journal of Experimental Psychology: Human Experimental Psychology, 51(A), 225-249.

Meegan, D. V., \& Tipper, S. P. (1999). Visual search and target-directed action. Journal of Experimental Psychology: Human Perception and Performance, 25, 1347-1362.

Meyer, D. E., Abrams, R. A., Kornblum, S., Wright, C. E., \& Smith, 
J. E. K. (1988). Optimality in human motor performance: Ideal control of rapid aimed movements. Psychological Review, 95, 340-370.

Miller, J. (1991). The flanker compatibility effect as a function of visual angle, attentional focus, visual transients, and perceptual load: A search for boundary conditions. Perception \& Psychophysics, 49, 270-288.

Pratt, J., \& Abrams, R. A. (1994). Action-centered inhibition: Effects of distractors on movement planning and execution. Human Movement Science, 13, 245-254.

Rosenbaum, D. A. (1991). Human motor control. San Diego, CA: Academic Press.

Simone, P. M., \& Baylis, G. C. (1997). Selective attention in a reaching task: Effect of normal aging and Alzheimer's disease. Journal of Experimental Psychology: Human Perception and Performance, 23, 595608.

Tipper, S. P., Howard, L. A., \& Houghton, G. (2000). Behavioural consequences of selection from neural population codes. In S. Monsell \& J. Driver (Eds.), Attention and performance XVII: Control of cognitive processes (pp. 223-245). Cambridge, MA: MIT Press.

Tipper, S. P., Howard, L. A., \& Jackson, S. R. (1997). Selective reaching to grasp: Evidence for distractor interference effects. Visual Cognition, $4,1-38$
Tipper, S. P., Howard, L. A., \& Paul, M. (2001). Reaching affects saccade trajectories. Experimental Brain Research, 136, 241-249.

Tipper, S. P., Lortie, C., \& Baylis, G. C. (1992). Selective reaching: Evidence for action-centered attention. Journal of Experimental Psychology: Human Perception and Performance, 18, 891-905.

Tipper, S. P., Weaver, B., \& Houghton, G. (1994). Behavioural goals determine inhibitory mechanisms of selective attention. Quarterly Journal of Experimental Psychology: Human Experimental Psychology, 47(A), 809-840.

Welsh, T. N., Elliott, D., \& Weeks, D. J. (1999). Hand deviations toward distractors: Evidence for response competition. Experimental Brain Research, 127, 207-212.

Woodin, M. E., \& Allport, A. (1998). Independent reference frames in human spatial memory: Body-centered and environment-centered coding in near and far space. Memory \& Cognition, 26, 1109-1116.

Yantis, S. (1992). Multielement visual tracking: Attention and perceptual organization. Cognitive Psychology, 24, 295-340.

Received October 9, 2000

Revision received July 24, 2001

Accepted July 30, 2001

\section{American Psychological Association SUBSCRIPTION ClaIMS INFORMATION}

Today's Date:

We provide this form to assist members, institutions, and nonmember individuals with any subscription problems. With the appropriate information we can begin a resolution. If you use the services of an agent, please do NOT duplicate claims through them and directly to us. PLEASE PRINT CLEARLY AND IN INK IF POSSIBLE.

\begin{tabular}{llll}
\hline PRINT FULL NAME OR KEY NAME OF INSTITUTION & & \\
\hline ADDRESS & & & \\
\hline CITY & & & \\
\hline
\end{tabular}

YOUR NAME AND PHONE NUMBER

\section{MEMBER OR CUSTOMER NUMBER (MAY BE FOUND ON ANY PAST ISSUE LABEL)}

DATE YOUR ORDER WAS MAIEDD (OR PHONED)

PREPAID _ CHECK____ CHARGE

CHECK/CARD CLEARED DATE:

(If possible, send a copy, front and back, of your cancelled check to help us in our research of your claim.)

ISSUES: ___ MISSING DAMAGED

TITLE

VOLUME OR YEAR

NUMBER OR MONTH

Thank you. Once a claim is received and resolved, delivery of replacement issues routinely takes 4-6 weeks.

(TO BE FILLED OUT BY APA STAFF)

DATE RECEIVED:

ACTION TAKEN:

DATE OF ACTION

ST AFF NAME:

INV. NO. \& DATE:

LABEL NO. \& DATE:

Send this form to APA Subscription Claims, 750 First Street, NE, Washington, DC 20002-4242

PLEASE DO NOT REMOVE. A PHOTOCOPY MAY BE USED. 